\title{
Design of Riverbank Riprap Using Large, Individually Placed Blocks
}

\author{
Mona Jafarnejad ${ }^{1}$; Mário J. Franca ${ }^{2}$; Michael Pfister ${ }^{3}$; and Anton J. Schleiss, M.ASCE ${ }^{4}$
}

\begin{abstract}
The protection of river banks in mountain rivers by riprap requires large blocks with weights typically exceeding $1 \mathrm{t}$. Thus, the blocks have to be placed individually. Such packed riprap has a relatively small spacing between the blocks. Consequently, the interlocking forces between the blocks are high, and the resistance of this protection to erosion is increased compared with traditional dumped riprap used for bank protection in low-grade rivers. Based on 98 systematical laboratory experiments, an adapted design method was developed for the case of riprap riverbank protection using large, individually placed blocks. Three different block sizes were tested by varying the longitudinal channel slope and bank inclination. For comparison, 34 tests were performed with dumped riprap. A design relationship is presented as a function of block size relative to the flow depth and a modified block Froude number considering the mean flow velocity. The additional resistance of riprap to erosion with large, individually placed blocks compared with dumped riprap is quantified. The proposed equations are compared with existing riprap sizing equations. The suggested method proved to be reliable in the assessment of the stability of packed riprap. Furthermore, it is shown that a second layer significantly delays riprap failure. DOI: 10.1061/(ASCE)HY.1943-7900.0001641. (C) 2019 American Society of Civil Engineers.
\end{abstract}

\section{Introduction}

The erodible banks of mountain rivers have to be protected against possible erosion in the vicinity of critical infrastructures. Among all flood protection measures ensuring river bank safety against lateral erosion, riprap is one of the most commonly used. Riprap has the advantage of offering flexible protection with self-healing capacity and a progressive failure mode. Furthermore, it has low construction costs and long-lasting performance, and it is easy to repair. Several methods of riprap design exist, which have been mostly developed for dumped blocks. However, in mountain rivers and steep channels, bank stability must be provided by using large blocks, individually placed by machinery due to their heavy weight typically exceeding $1 \mathrm{t}$ (Schleiss 2000). The term block refers to large rock blocks extracted from quarries having sharp edges that increase the stability of a riprap consisting of individually placed large blocks. The blocks are consequently better packed and interlocked with small joints between them compared with dumped riprap.

${ }^{1}$ Researcher, Laboratory of Hydraulic Constructions, École Polytechnique, Fédérale de Lausanne, 1015 Lausanne, Switzerland (corresponding author). ORCID: https://orcid.org/0000-0003-0087-1247. Email: mona .jafarnejad@alumni.epfl.ch

${ }^{2}$ Professor, Dept. of Water Science and Engineering, IHE Delft Institute for Water Education, Westvest 7, 2611 AX, Delft, Netherlands; Dept. of Hydraulic Engineering, Delft Univ. of Technology, 2601 DA, Delft, Netherlands. Email: m.franca@un-ihe.org

${ }^{3}$ Professor, Dept. of Civil Engineering, Haute Ecole d'Ingénierie et d'Architecture, Univ. of Applied Sciences and Arts Western Switzerland, CH-1705 Fribourg, Switzerland. Email: michael.pfister@hefr.ch

${ }^{4}$ Professor Emeritus, Laboratory of Hydraulic Constructions, École Polytechnique Fédérale de Lausanne, 1015 Lausanne, Switzerland. Email: anton.schleiss@epfl.ch

Note. This manuscript was submitted on September 13, 2018; approved on March 29, 2019; published online on September 30, 2019. Discussion period open until February 29, 2020; separate discussions must be submitted for individual papers. This paper is part of the Journal of Hydraulic Engineering, (C) ASCE, ISSN 0733-9429.
Riprap construction by machine is shown in Fig. 1, where large blocks are separately placed on a geotextile. The additional stability of a well-packed riprap with individually placed large blocks is not known. Several equations have been developed for dumped riprap to predict its stability when exposed to a river flow, considering block sizes, gradation of the blocks, and thickness of the riprap as geometrical parameters, as well as the characteristics of an underlying filter (Stevens et al. 1976; Maynord et al. 1991; Maynord and Neil 2008; Escarameia and May 1992).

Most riprap design methods are based on those of Shields (1936) (Recking and Pitlick 2013) and Isbash (1935), who performed tests on the entrainment of particles subject to changing shear stress and velocity. In particular, the velocity-based method of Isbash (1935) was one of the first directly adapted to riprap design. He analyzed the stability of riprap exposed to a river flow and defined a critical flow velocity that would move a block based on the block diameter. The main purpose of his tests was to develop criteria for the stability of overflown cofferdams; however, the equation was later used for riprap design (USACE 1990). Lane (1952) used the shear-stress method for stable canal design with noncohesive material. Anderson et al. (1970) enhanced this method and included the effects of bank slopes and channel bends. Li et al. (1976) and Simons and Senturk (1992) developed a method based on tractive force that allows the determination of safety factors.

The stability of loose (dumped) rock riprap against erosive forces due to flowing water was also studied by Froehlich (2013). His evaluation was based on the ratio of static moments resisting the overturning of the blocks. The moment ratio is defined as a safety factor that indicates the potential failure. Abt et al. (2008) studied the stability of rounded riprap subjected to overtopping flow. The concept of a safety factor has been introduced by several researchers to riprap design. Stevens et al. (1976) presented a safety factor by taking into account the stability of an individual block in the riprap. Each block remains stable if the sum of the moments causing the possible displacement of a block is less than the moment due to the submerged weight. Froehlich (2013), Ulrich (1987), and Stevens et al. (1984) also considered the weight of the submerged block as the only resisting force. Wittler and Abt (1988) 


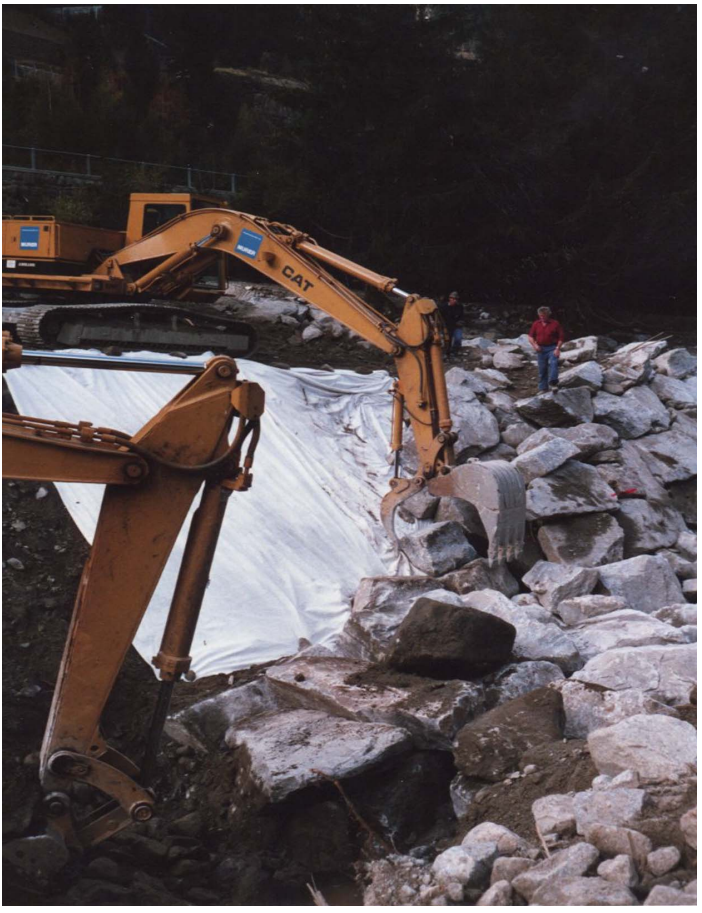

Fig. 1. Riprap with blocks of $4-4.5$ t being individually placed by a machine on a geotextile serving as a filter. (Image by A. J. Schleiss.)

modified Stevens et al.'s (1976) analysis by adding contact and frictional forces from nearby blocks.

Froehlich and Benson (1996) worked on a wide range of angle of reposes to consider the effect of bank inclination on riprap stability by proposing a "particle angle of initial yield." Escarameia and May (1992) gave a general equation for designing riverbank protection by ripraps and gabion mattresses. Brown and Clyde (1989) used both the Manning-Strickler equation with the Shields relation to make a combined equation for defining the size of blocks that remain stable. Similar approaches were given earlier by Straub (1953), Grace et al. (1973), and Reese (1984).

Maynord (1992) performed the same experiments with doublelayer riprap to evaluate the thickness effect and reported it as the stability criteria. For relatively low-turbulence applications such as riverbank protections, USACE (1994) specified a minimum thickness of the riprap based on $D_{100}$ or $1.5 D_{50}$, whichever is greater. The stability tests of Abt and Johnson (1991) and Maynord (1992) revealed that any additional thickness above these minimum values results in a higher stability. Consequently, a larger thickness of a smaller gradation may sometimes provide equivalent stability. The increase in stability with riprap thickness is more significant for wide block gradations but relatively low for uniform gradations (Maynord and Neill 2008). The improvement of the stability with increasing thickness can be explained by the fact that more protection material is available, which can move to damaged areas and cover them again. More energy is then dissipated to expose the filter underlying the blocks, which means that the riprap failure will eventually be postponed.

The aforementioned design methods are limited to dumped blocks, and the first movement of a block is used as failure criterion when the underlying layer is exposed to the flow (De Almeida and Martín-Vide 2009; Hiller et al. 2017). However, if large blocks are needed for stability reasons in mountain rivers, they have to be placed individually by a machine due to their heavy weight. Jafarnejad et al. (2017b) studied the effect of large blocks individually placed as riprap and estimated the time to failure of such riprap. The behavior of individually placed large blocks as riprap bank protection was systematically studied with laboratory experiments. Ninety-eight experiments were performed in an open channel with three different longitudinal channel slopes of $1.5 \%$, $3 \%$, and $5.5 \%$ and three riprap bank inclinations of $35^{\circ}, 31^{\circ}$, and $27^{\circ}$. Furthermore, 34 tests with dumped riprap were performed for comparison with existing riprap design methods. Finally, the effect of the riprap thickness (i.e., number of layers) was assessed with an additional 21 double-layer tests of packed riprap.

\section{Comparison of the Design Equations for Dumped Riprap}

In general, riprap protection is endangered by the erosive effects of drag and lift forces resulting from the velocity distribution near the blocks. The basic form of many design equations for obtaining the required block diameter has been given by Maynord and Neill (2008)

$$
\frac{V_{C}}{\left[g\left(S_{B}-1\right) D\right]^{\frac{1}{2}}}=C_{\text {ref }}\left(\frac{D}{h}\right)^{P_{\text {ref }}}
$$

where $V_{c}=$ characteristic flow velocity $\left(\mathrm{ms}^{-1}\right) ; g=$ acceleration of gravity $\left(\mathrm{ms}^{-2}\right) ; S_{B}=$ specific gravity of the block, $S_{B}=\rho_{s} / \rho$, where $\rho_{s}$ is the block density $\left(\mathrm{kgm}^{-3}\right)$ and $\rho$ is the water density $\left(\mathrm{kgm}^{-3}\right) ; D=$ characteristic particle size $(\mathrm{m}) ; C_{\text {ref }}=$ numerical coefficient, usually based on empirical data; $h=$ local flow depth; and $P_{\text {ref }}=$ exponent dependent on the hydraulic conditions and on the definition of the characteristic velocity $V_{c}\left(\mathrm{~ms}^{-1}\right)$.

Among the numerous design equations for bank protection by riprap, the following are the most frequently used in practice: (1) Hydraulic Engineering Circular No. 11 [HEC-11 (Brown and Clyde 1989)], (2) Escarameia and May (1992), (3) Pilarczyk (1990), (4) Engineering Manual No. 1601 [EM-1601 (USACE 1994)] supplemented by Maynord et al. (1989) and Maynord (1992), (5) Isbash (1935), (6) California Bank and Shore Rock slope design [CABS (Racin et al. 2000)], and (7) Hydraulic Design Series No. 6 [HDS 6 (Richardson and Davis 2001)].

These seven design equations have been compared with data from a study of three sites by Blodgett and McConaughy (1986) based on the report by Lagasse et al. (2006). Two of these equations were selected for further analyses and comparisons: EM-1601 is the most comprehensive, and HEC-11 is the equation that is frequently applied (Lagasse et al. 2006). Lagasse et al. (2006) reduced these equations to their basic form by removing the correction factors related to the bend radius and safety/stability factors. Furthermore, they converted each equation to its dimensionless form. The equations serve to estimate the riprap size for a straight channel and for incipient motion conditions. They are considered for a bank inclination of $35^{\circ}$, and all have the same dependent and independent variables. The differences are related to the coefficient and exponent applied at the independent variable. HEC-11 results in the smallest block size considering that they refer to $D_{50}$, whereas EM-1601 considers $D_{30}$ (Maynord 1992).

\section{Experimental Setup and Procedure}

The laboratory tests were carried out in a straight $10-\mathrm{m}$ reach of a 23 -m-long and 1.5-m-wide tilting flume (Fig. 2). The longitudinal slope of the flume was set to $S=1.5 \%, 3 \%$, and $5.5 \%$. The tested riprap bank inclinations were $\alpha=35^{\circ}(1.43 \mathrm{H}: 1 \mathrm{~V})$, $31^{\circ}(1.66 \mathrm{H}: 1 \mathrm{~V})$ and $27^{\circ}(2 \mathrm{H}: 1 \mathrm{~V})$. Only half of the trapezoidal 


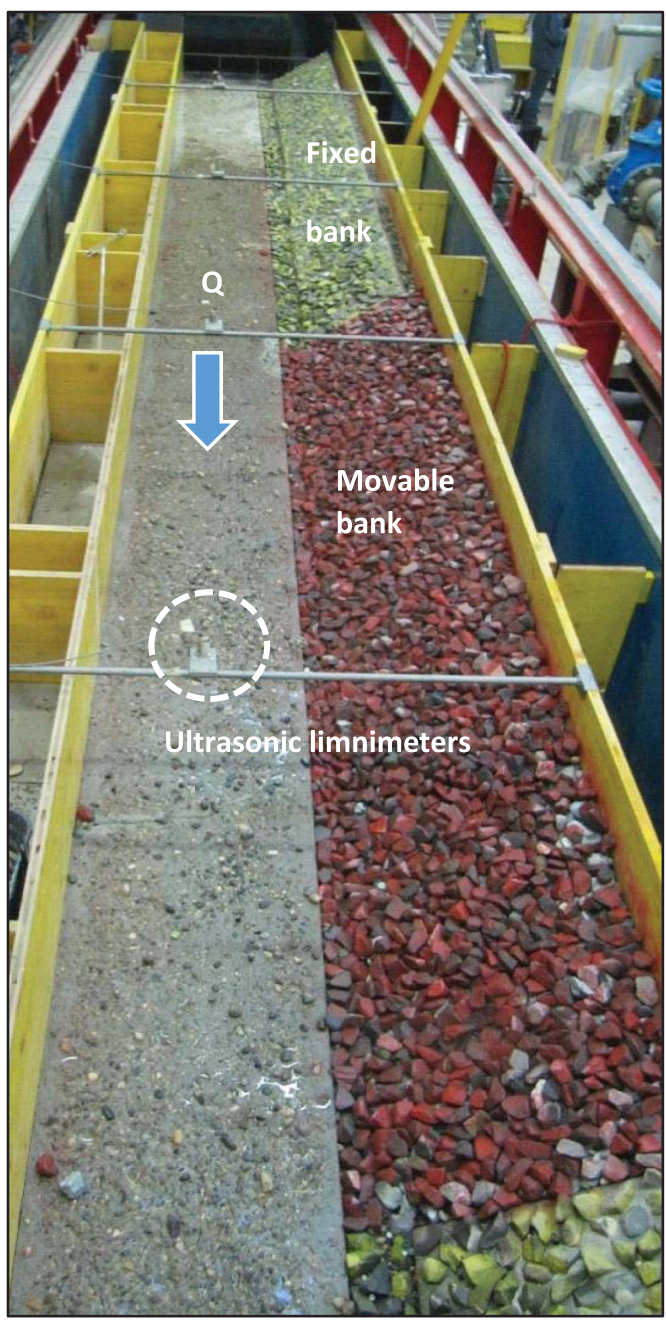

Fig. 2. Experimental flume (seen against the flow direction) with riprap on the left bank (half-symmetrical part of the riverbed).

channel was reproduced assuming symmetrical conditions. The scaling parameters, under Froude similarity, are water depth and time to failure. A geometrical scale factor of the laboratory tests compared to typical mountain river applications is approximately $1: 20-1: 30$

The block shape has an influence on the achievable packing of the riprap and thus on its stability and resistance against erosion. In general, the shape of the blocks may change from tabular and irregular blocks to equant, half-rounded, and rounded blocks. Studies have shown that irregular blocks with sharp edges from quarries are more stable than equant rocks and much more stable than blocks that have been rounded as a result of river transport. The last two block shapes are inappropriate for riprap (USBR 2014). Large blocks used for large, individually placed riprap in mountain rivers have to be extracted by blasting from quarries. For the experiments, irregular crushed stones with sharp edges were used. They were categorized into three classes based on their weight, namely Class I of $0.050-0.090 \mathrm{~kg}$, Class II of $0.090-0.130 \mathrm{~kg}$, and Class III of $0.130-0.170 \mathrm{~kg}$ (Fig. 3). Based on a specific gravity of 2.66 for the crushed stones, their equivalent diameter for a spherical block was calculated for Class I with $D_{50}=0.037 \mathrm{~m} \pm 9.6 \%$ (range of block sizes between $D_{\min }$ and $D_{\max }$ ), Class II with $D_{50}=$ $0.042 \mathrm{~m} \pm 4.7 \%$, and Class III with $D_{50}=0.047 \mathrm{~m} \pm 4.2 \%$, as given in Table 1 .

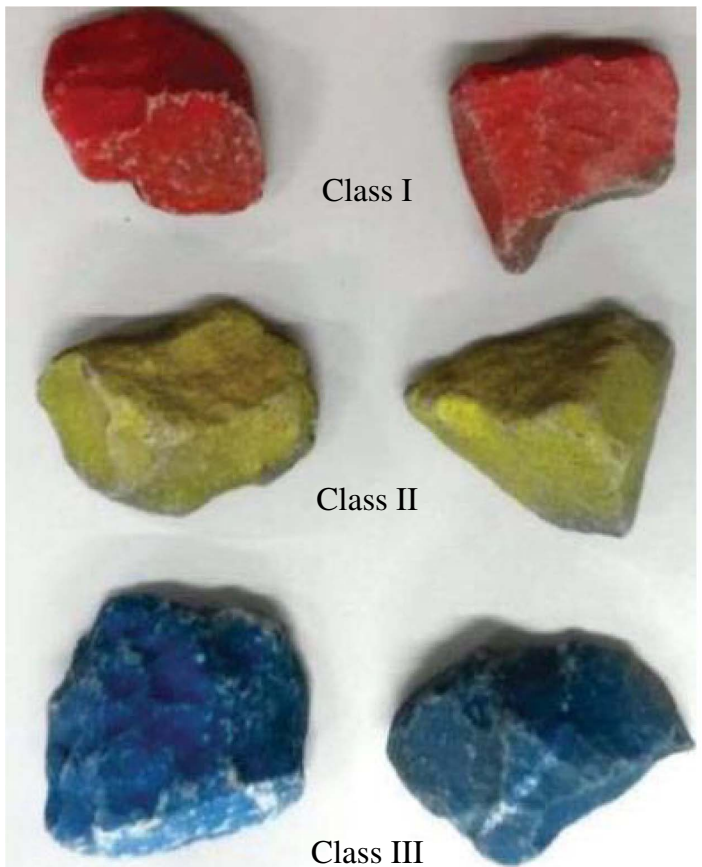

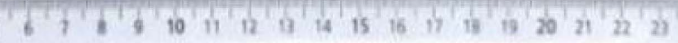

Fig. 3. Three block classes defined as Class $\mathrm{I}=0.050-0.090 \mathrm{~kg}$ $\left(D_{50}=0.037 \mathrm{~m}\right)$, Class II $=0.090-0.130 \mathrm{~kg}\left(D_{50}=0.042 \mathrm{~m}\right)$, and Class III $=0.130-0.170 \mathrm{~kg}\left(D_{50}=0.047 \mathrm{~m}\right)$.

The three block classes have relatively uniform grain-size distributions for practical applications. Ahmed (1987) and Wittler and Abt (1988) reported that a riprap with a uniform gradation (when $\sigma$ is close to 1 ) tends to be more stable compared with a wide distribution of block sizes. The obtained angle of repose of the blocks used in the experiments is between $40^{\circ}$ and $41^{\circ}$ [according to Froehlich (2013) and Jafarnejad et al. (2017a)]. Fig. 4 shows the cross section of the experimental flume with a riprap of one and two layers of blocks. The arrangement of the packed blocks is shown in Fig. 5(a). The blocks were placed and packed on river bank material with a large grain-size distribution as given in Table $2(\sigma=3.75)$ satisfying filter criteria [Fig. 5(a)]. To simulate natural hydraulic roughness conditions, the fixed material of the channel bed with mortar was the same as that used in the loose banks underneath the riprap $\left(D_{50}=\right.$ $0.0053 \mathrm{~m})$.

The distinction between dumped and well-packed riprap was based on porosity measurements. Preliminary tests were performed to obtain the void volume in a defined dumped or packed riprap volume. Theresults showed that the porosity of the packed riprap was reduced by $2 \%$ (from $43.71 \%$ to $41.52 \%$ ) for $D_{50}=$ $0.037 \mathrm{~m}$, by $4 \%$ (from $44.73 \%$ to $40.78 \%$ ) for $D_{50}=0.042 \mathrm{~m}$, and by $10 \%$ (from $46.56 \%$ to $36.8 \%$ ) for $D_{50}=0.047 \mathrm{~m}$ when compared with the dumped riprap (Jafarnejad et al. 2017a).

The corresponding prototype time is important for the analysis of the results because it represents the expected maximum duration of the flood peak assuming a constant mean discharge, which may cause riprap failure. The time scale of the physical model based on the Froude similarity is given 
Table 1. Characteristics of the blocks used as riprap

\begin{tabular}{lcccccccccc}
\hline Class & Size $(\mathrm{m})$ and weight $(\mathrm{kg})$ & $D_{\min }$ & $D_{15}$ & $D_{30}$ & $D_{50}$ & $D_{65}$ & $D_{85}$ & $D_{\max }$ & $\sigma\left(D_{85} / D_{15}\right)$ \\
\hline I & Weight & 0.050 & 0.057 & 0.065 & 0.073 & 0.077 & 0.083 & 0.090 \\
& Size & 0.032 & 0.033 & 0.035 & 0.037 & 0.038 & 0.039 & 0.040 & 1.18 \\
II & Weight & 0.090 & 0.093 & 0.100 & 0.107 & 0.115 & 0.0123 & 0.130 \\
& Size & 0.040 & 0.041 & 0.0415 & 0.042 & 0.043 & 0.044 & 0.045 & 1.07 \\
III & Weight & 0.130 & 0.135 & 0.143 & 0.147 & 0.158 & 0.163 & 0.170 & 1.06 \\
& Size & 0.045 & 0.046 & 0.0465 & 0.047 & 0.048 & 0.049 & 0.05 \\
\hline
\end{tabular}
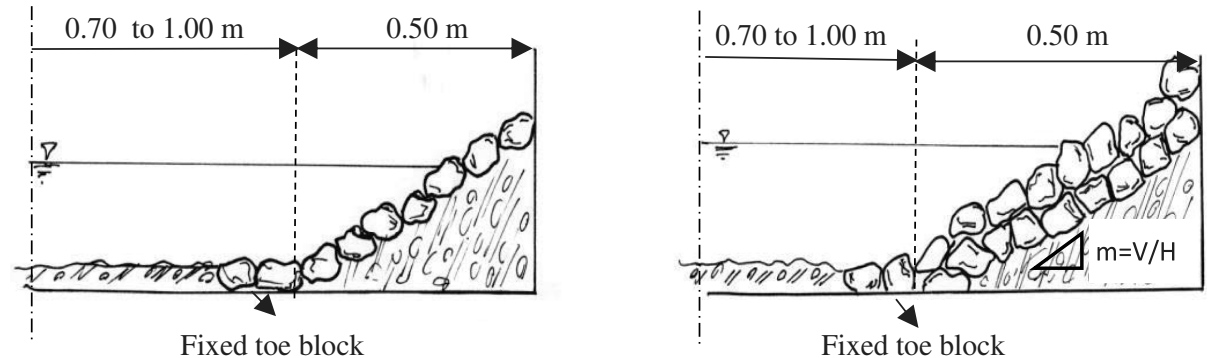

Fig. 4. Cross section of the experimental flume with one and two layers of blocks. Bank inclination is defined as $V: H$, where $V$ stands for vertical and $H$ stands for horizontal values.

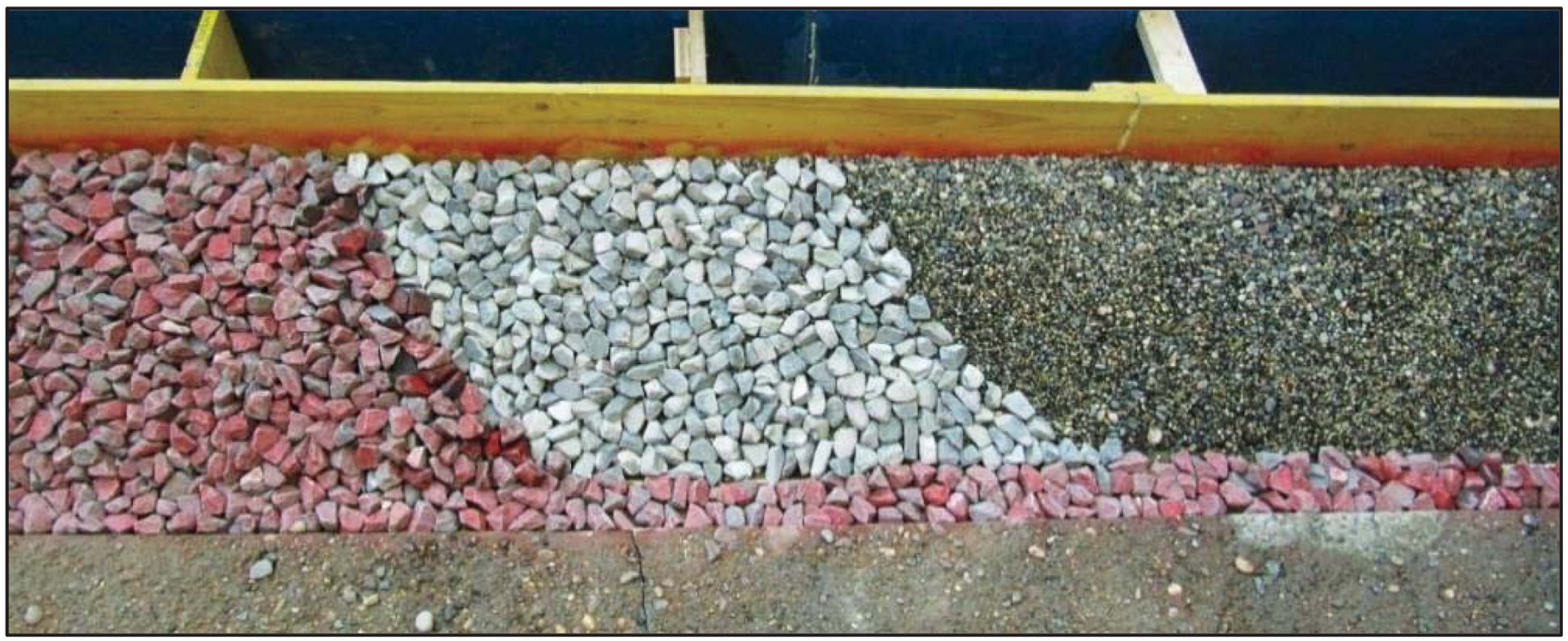

(a)

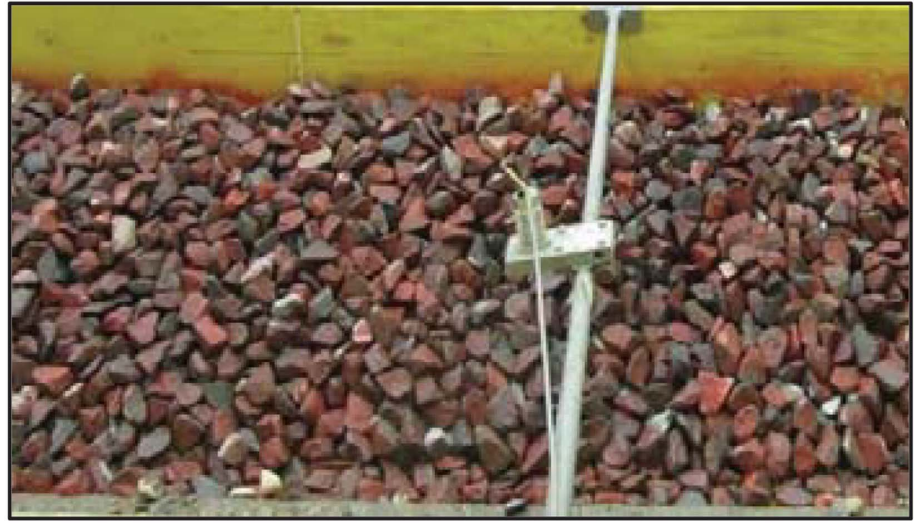

(b)

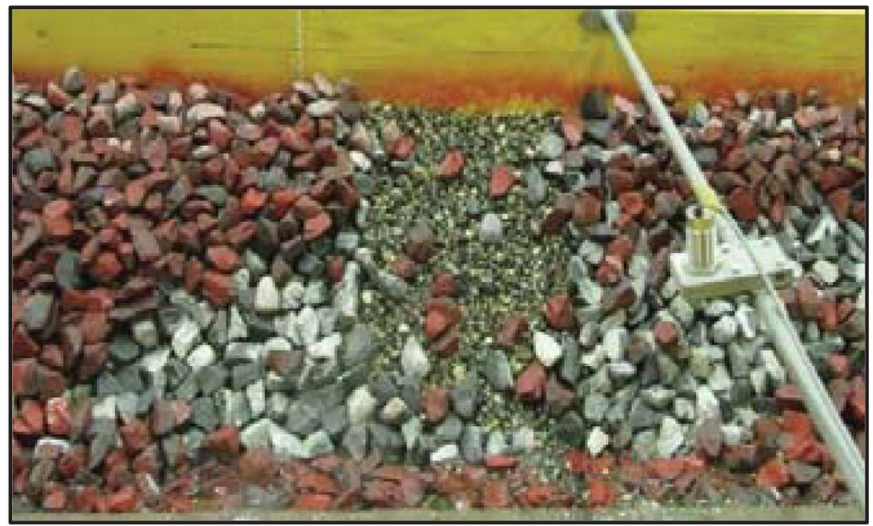

(c)

Fig. 5. Experimental setup of a two-layer test showing (a) installation of the two layers of riprap; and test Double 8 (Table 5); (b) before; and (c) after the complete failure of the riprap. 


$$
\lambda_{T}=\frac{T_{p}}{T_{m}}=\sqrt{\frac{L_{p}}{L_{m}}}
$$

where $T=$ duration; $L=$ length; and subscripts $p$ and $m=$ prototype and model, respectively.

Considering a geometrical scale of the experimental setup for typical alpine mountain rivers of $L_{P} / L_{m}=25$, as mentioned previously, the corresponding time scale is $\lambda_{T}=5$. Thus, laboratory tests lasting $180 \mathrm{~min}$ are roughly equivalent to a prototype flood peak duration of approximately $15 \mathrm{~h}$. The statistics of typical floods in alpine rivers reveal that the peak region of the flood hydrograph

Table 2. Characteristics of the sediments corresponding to the bank filter underneath the riprap, which are the same glued to the channel bed to form a rough bed

\begin{tabular}{lr}
\hline$D_{x}$ & Size \\
\hline$D_{10}(\mathrm{~mm})$ & 3.2 \\
$D_{35}(\mathrm{~mm})$ & 4.4 \\
$D_{50}(\mathrm{~mm})$ & 5.3 \\
$D_{m}=D_{65}(\mathrm{~mm})$ & 8.5 \\
$D_{75}(\mathrm{~mm})$ & 9.1 \\
$D_{90}(\mathrm{~mm})$ & 14.8 \\
$D_{\max }(\mathrm{mm})$ & 32 \\
$D_{60} / D_{10}$ & 2.5 \\
\hline
\end{tabular}

Note: $D_{m}=$ mean diameter and corresponds to $D_{65}$. rarely exceeds a duration of $15 \mathrm{~h}$ for extreme events (Bezzola and Hegg 2007, 2008). Therefore, the maximum duration of the experiments was set to $180 \mathrm{~min}$ based on preliminary tests and considering the range of flood peak durations in mountain rivers. Total bank failure is defined as soon as all blocks have been washed away or slid down vertically in a determined cross section. The erosion of single blocks in a packed riprap was not considered as a total failure.

The discharge was provided by the laboratory pump equipped with an electromagnetic flowmeter [ABB-FXE4000-COPA (ABB, Zurich, Switzerland)] with a precision of $\pm 0.5 \%$. The discharge was increased successively to identify the stability limit of the riprap and the corresponding discharge that causes failure. Tables 3-5 present the characteristics of the block material and the detailed test program. Lower discharges could cause only some direct erosion of single blocks but not lead to full failure of the riprap over the whole bank height. For the tested channel slopes, transcritical or supercritical flow conditions $(F r>0.90)$ occurred for all discharges (Table 4). The discharge was subsequently increased (for following tests) until total failure occurred, providing the failure discharge.

The flow depths were measured by ultrasonic distance measuring sensors (Baumer UNAM 30I6103/S14, Baumer, Frauenfeld, Switzerland) with a precision of $0.5 \%$ at four different positions along the observation reach at 2-m intervals along the channel axis. They were all transversally located at the center of each section. In the first upstream reach of $6.5 \mathrm{~m}$, the riprap was fixed with mortar while maintaining the same roughness to avoid any influence of the model inlet. The observation of the block erosion was limited to the

Table 3. Tests with dumped riprap, $S=0.03$, and $\alpha=35^{\circ}$

\begin{tabular}{|c|c|c|c|c|c|c|c|c|}
\hline Test series & $D_{50}(\mathrm{~m})$ & $Q\left(\mathrm{~m}^{3} \mathrm{~s}^{-1}\right)$ & $h(\mathrm{~m})$ & $A\left(\mathrm{~m}^{2}\right)$ & $R_{h}(\mathrm{~m})$ & $V\left(\mathrm{~ms}^{-1}\right)$ & $\mathrm{Fr}$ & Failed or stable \\
\hline D1.1 & 0.037 & 0.013 & 0.018 & 0.02 & 0.017 & 0.63 & 1.49 & $\mathrm{~S}$ \\
\hline D1.2 & 0.037 & 0.023 & 0.028 & 0.03 & 0.027 & 0.74 & 1.41 & S \\
\hline D1.3 & 0.037 & 0.056 & 0.050 & 0.05 & 0.046 & 1.08 & 1.55 & S \\
\hline D1.4 & 0.037 & 0.077 & 0.057 & 0.06 & 0.051 & 1.23 & 1.64 & S-F \\
\hline D1.5 & 0.037 & 0.095 & 0.064 & 0.07 & 0.057 & 1.30 & 1.64 & $\mathrm{~F}$ \\
\hline D1.6 & 0.037 & 0.111 & 0.072 & 0.08 & 0.063 & 1.32 & 1.57 & $\mathrm{~F}$ \\
\hline D1.7 & 0.037 & 0.138 & 0.095 & 0.10 & 0.080 & 1.29 & 1.34 & $\mathrm{~F}$ \\
\hline D1.8 & 0.037 & 0.161 & 0.100 & 0.11 & 0.084 & 1.36 & 1.38 & $\mathrm{~F}$ \\
\hline D1.9 & 0.037 & 0.170 & 0.105 & 0.11 & 0.088 & 1.44 & 1.41 & $\mathrm{~F}$ \\
\hline D1.10 & 0.037 & 0.194 & 0.109 & 0.12 & 0.090 & 1.50 & 1.45 & $\mathrm{~F}$ \\
\hline D1.11 & 0.037 & 0.203 & 0.113 & 0.12 & 0.093 & 1.57 & 1.49 & $\mathrm{~F}$ \\
\hline D1.12 & 0.037 & 0.227 & 0.116 & 0.13 & 0.095 & 1.61 & 1.51 & $\mathrm{~F}$ \\
\hline D1.13 & 0.037 & 0.237 & 0.118 & 0.13 & 0.097 & 1.68 & 1.56 & F \\
\hline D1.14 & 0.037 & 0.253 & 0.126 & 0.14 & 0.102 & 1.66 & 1.49 & F \\
\hline D1.15 & 0.037 & 0.271 & 0.126 & 0.14 & 0.102 & 1.78 & 1.60 & F \\
\hline D1.16 & 0.037 & 0.325 & 0.152 & 0.17 & 0.119 & 1.73 & 1.41 & $\mathrm{~F}$ \\
\hline D1.17 & 0.037 & 0.369 & 0.170 & 0.19 & 0.130 & 1.73 & 1.34 & F \\
\hline D2.1 & 0.042 & 0.006 & 0.012 & 0.012 & 0.012 & 0.48 & 1.41 & $S$ \\
\hline D2.2 & 0.042 & 0.021 & 0.026 & 0.026 & 0.025 & 0.79 & 1.56 & $S$ \\
\hline D2.3 & 0.042 & 0.051 & 0.045 & 0.046 & 0.041 & 1.08 & 1.62 & S \\
\hline D2.4 & 0.042 & 0.067 & 0.053 & 0.055 & 0.048 & 1.17 & 1.63 & $S$ \\
\hline $\mathrm{D} 2.5$ & 0.042 & 0.086 & 0.062 & 0.065 & 0.055 & 1.27 & 1.63 & $S$ \\
\hline D2.6 & 0.042 & 0.109 & 0.072 & 0.076 & 0.063 & 1.37 & 1.62 & S-F \\
\hline D2.7 & 0.042 & 0.142 & 0.086 & 0.091 & 0.074 & 1.47 & 1.60 & F \\
\hline D2.8 & 0.042 & 0.155 & 0.091 & 0.097 & 0.078 & 1.50 & 1.58 & F \\
\hline D2.9 & 0.042 & 0.173 & 0.098 & 0.105 & 0.083 & 1.54 & 1.57 & F \\
\hline D2.10 & 0.042 & 0.188 & 0.104 & 0.112 & 0.087 & 1.57 & 1.55 & $\mathrm{~F}$ \\
\hline D2.11 & 0.042 & 0.208 & 0.111 & 0.120 & 0.092 & 1.60 & 1.53 & F \\
\hline D2.12 & 0.042 & 0.224 & 0.117 & 0.127 & 0.096 & 1.62 & 1.51 & F \\
\hline D2.13 & 0.042 & 0.233 & 0.121 & 0.131 & 0.099 & 1.63 & 1.50 & F \\
\hline D2.14 & 0.042 & 0.251 & 0.127 & 0.139 & 0.103 & 1.65 & 1.48 & F \\
\hline D2.15 & 0.042 & 0.251 & 0.127 & 0.139 & 0.103 & 1.65 & 1.48 & F \\
\hline D2.16 & 0.042 & 0.325 & 0.154 & 0.171 & 0.120 & 1.71 & 1.39 & F \\
\hline D2.17 & 0.042 & 0.375 & 0.172 & 0.193 & 0.131 & 1.73 & 1.33 & F \\
\hline
\end{tabular}

Note: $\mathrm{F}=$ failed test; $\mathrm{S}=$ stable test; and $\mathrm{F}-\mathrm{S}=$ transitional test. 
Table 4. Test program with packed riprap (individually placed blocks)

\begin{tabular}{|c|c|c|c|c|c|c|c|c|c|}
\hline $\begin{array}{l}\text { Test } \\
\text { series }\end{array}$ & $\begin{array}{l}\text { Channel } \\
\text { slope, } S\end{array}$ & $\begin{array}{c}\text { Bank inclination, } \\
\alpha \text { (degrees) }\end{array}$ & $D_{50}(\mathrm{~m})$ & $h(\mathrm{~m})$ & $Q\left(\mathrm{~m}^{3} \mathrm{~s}^{-1}\right)$ & $V\left(\mathrm{~ms}^{-1}\right)$ & $F r$ & $\tau(\mathrm{Pa})$ & $\begin{array}{c}\text { Failed or } \\
\text { stable } \\
\end{array}$ \\
\hline 1 & 0.015 & 35 & 0.047 & 0.240 & 0.35 & 1.673 & 1.09 & 24.96 & $S$ \\
\hline 2 & 0.015 & 35 & 0.047 & 0.252 & 0.38 & 1.714 & 1.09 & 25.91 & S \\
\hline 3 & 0.015 & 35 & 0.047 & 0.290 & 0.40 & 1.520 & 0.90 & 28.80 & F-S \\
\hline 4 & 0.015 & 35 & 0.047 & 0.295 & 0.42 & 1.563 & 0.92 & 29.17 & $\mathrm{~F}$ \\
\hline 5 & 0.015 & 35 & 0.047 & 0.310 & 0.50 & 1.743 & 1.00 & 30.27 & $\mathrm{~F}$ \\
\hline 6 & 0.015 & 35 & 0.037 & 0.180 & 0.23 & 1.542 & 1.16 & 19.96 & S \\
\hline 7 & 0.015 & 35 & 0.037 & 0.187 & 0.24 & 1.540 & 1.14 & 20.57 & S \\
\hline 8 & 0.015 & 35 & 0.037 & 0.197 & 0.26 & 1.540 & 1.11 & 21.43 & F-S \\
\hline 9 & 0.015 & 35 & 0.037 & 0.202 & 0.27 & 1.554 & 1.10 & 21.85 & $\mathrm{~F}$ \\
\hline 10 & 0.015 & 35 & 0.037 & 0.209 & 0.28 & 1.582 & 1.11 & 22.40 & $\mathrm{~F}$ \\
\hline 11 & 0.015 & 35 & 0.037 & 0.215 & 0.30 & 1.607 & 1.11 & 22.94 & $\mathrm{~F}$ \\
\hline 12 & 0.015 & 35 & 0.042 & 0.222 & 0.33 & 1.731 & 1.17 & 23.51 & $\mathrm{~S}$ \\
\hline 13 & 0.015 & 35 & 0.042 & 0.230 & 0.34 & 1.710 & 1.14 & 24.16 & S \\
\hline 14 & 0.015 & 35 & 0.042 & 0.239 & 0.35 & 1.682 & 1.10 & 24.88 & F-S \\
\hline 15 & 0.015 & 35 & 0.042 & 0.246 & 0.37 & 1.718 & 1.11 & 25.44 & $\mathrm{~F}$ \\
\hline 16 & 0.015 & 35 & 0.042 & 0.250 & 0.38 & 1.730 & 1.11 & 25.75 & $\mathrm{~F}$ \\
\hline 17 & 0.015 & 35 & 0.042 & 0.259 & 0.41 & 1.767 & 1.11 & 26.45 & $\mathrm{~F}$ \\
\hline 18 & 0.030 & 35 & 0.047 & 0.185 & 0.32 & 2.053 & 1.52 & 40.79 & $\mathrm{~S}$ \\
\hline 19 & 0.030 & 35 & 0.047 & 0.187 & 0.33 & 2.085 & 1.54 & 41.13 & S \\
\hline 20 & 0.030 & 35 & 0.047 & 0.202 & 0.34 & 1.994 & 1.42 & 43.70 & F-S \\
\hline 21 & 0.030 & 35 & 0.047 & 0.210 & 0.36 & 1.989 & 1.39 & 45.04 & $\mathrm{~F}$ \\
\hline 22 & 0.030 & 35 & 0.047 & 0.220 & 0.38 & 2.005 & 1.37 & 46.69 & $\mathrm{~F}$ \\
\hline 23 & 0.030 & 35 & 0.047 & 0.240 & 0.41 & 1.960 & 1.28 & 49.92 & $\mathrm{~F}$ \\
\hline 24 & 0.030 & 35 & 0.037 & 0.122 & 0.23 & 1.719 & 1.57 & 30.71 & $\mathrm{~S}$ \\
\hline 25 & 0.030 & 35 & 0.037 & 0.134 & 0.27 & 1.818 & 1.59 & 33.30 & $\mathrm{~S}$ \\
\hline 26 & 0.030 & 35 & 0.037 & 0.136 & 0.28 & 1.843 & 1.60 & 33.73 & $\mathrm{~S}$ \\
\hline 27 & 0.030 & 35 & 0.037 & 0.141 & 0.29 & 1.869 & 1.59 & 34.78 & F-S \\
\hline 28 & 0.030 & 35 & 0.037 & 0.145 & 0.31 & 1.937 & 1.63 & 35.62 & $\mathrm{~F}$ \\
\hline 29 & 0.030 & 35 & 0.037 & 0.155 & 0.34 & 1.952 & 1.58 & 37.69 & $\mathrm{~F}$ \\
\hline 30 & 0.030 & 35 & 0.037 & 0.167 & 0.35 & 1.872 & 1.46 & 40.13 & $\mathrm{~F}$ \\
\hline 31 & 0.030 & 35 & 0.042 & 0.160 & 0.25 & 1.919 & 1.53 & 36.33 & $\mathrm{~S}$ \\
\hline 32 & 0.030 & 35 & 0.042 & 0.169 & 0.28 & 1.983 & 1.54 & 37.96 & $\mathrm{~S}$ \\
\hline 33 & 0.030 & 35 & 0.042 & 0.177 & 0.30 & 2.030 & 1.54 & 39.38 & F-S \\
\hline 34 & 0.030 & 35 & 0.042 & 0.181 & 0.31 & 2.052 & 1.54 & 40.09 & $\mathrm{~F}$ \\
\hline 35 & 0.030 & 35 & 0.042 & 0.183 & 0.32 & 2.072 & 1.55 & 40.44 & $\mathrm{~F}$ \\
\hline 36 & 0.030 & 35 & 0.042 & 0.187 & 0.33 & 2.085 & 1.54 & 41.13 & $\mathrm{~F}$ \\
\hline 37 & 0.030 & 35 & 0.042 & 0.152 & 0.33 & 1.929 & 1.58 & 37.08 & $\mathrm{~F}$ \\
\hline 38 & 0.030 & 35 & 0.042 & 0.156 & 0.35 & 1.990 & 1.61 & 37.90 & $\mathrm{~F}$ \\
\hline 39 & 0.030 & 35 & 0.042 & 0.205 & 0.35 & 2.017 & 1.42 & 44.20 & $\mathrm{~F}$ \\
\hline 40 & 0.030 & 31 & 0.047 & 0.172 & 0.28 & 1.930 & 1.49 & 39.80 & $\mathrm{~S}$ \\
\hline 41 & 0.030 & 31 & 0.047 & 0.179 & 0.30 & 1.974 & 1.49 & 41.13 & $\mathrm{~S}$ \\
\hline 42 & 0.030 & 31 & 0.047 & 0.196 & 0.35 & 2.080 & 1.50 & 44.28 & F-S \\
\hline 43 & 0.030 & 31 & 0.047 & 0.205 & 0.38 & 2.134 & 1.51 & 45.92 & $\mathrm{~F}$ \\
\hline 44 & 0.030 & 31 & 0.047 & 0.211 & 0.40 & 2.164 & 1.51 & 47.00 & $\mathrm{~F}$ \\
\hline 45 & 0.030 & 31 & 0.037 & 0.120 & 0.15 & 1.563 & 1.44 & 29.43 & $\mathrm{~S}$ \\
\hline 46 & 0.030 & 31 & 0.037 & 0.126 & 0.17 & 1.656 & 1.49 & 30.68 & $\mathrm{~S}$ \\
\hline 47 & 0.030 & 31 & 0.037 & 0.134 & 0.19 & 1.719 & 1.50 & 32.32 & F-S \\
\hline 48 & 0.030 & 31 & 0.037 & 0.147 & 0.23 & 1.886 & 1.57 & 34.94 & $\mathrm{~F}$ \\
\hline 49 & 0.030 & 31 & 0.037 & 0.160 & 0.27 & 1.995 & 1.59 & 37.49 & $\mathrm{~F}$ \\
\hline 50 & 0.030 & 31 & 0.042 & 0.145 & 0.24 & 1.974 & 1.66 & 34.54 & $\mathrm{~S}$ \\
\hline 51 & 0.030 & 31 & 0.042 & 0.158 & 0.25 & 1.903 & 1.53 & 37.10 & $\mathrm{~S}$ \\
\hline 52 & 0.030 & 31 & 0.042 & 0.165 & 0.27 & 1.918 & 1.51 & 38.46 & $\mathrm{~S}$ \\
\hline 53 & 0.030 & 31 & 0.042 & 0.176 & 0.30 & 1.980 & 1.51 & 40.56 & F-S \\
\hline 54 & 0.030 & 31 & 0.042 & 0.180 & 0.31 & 1.996 & 1.50 & 41.28 & $\mathrm{~F}$ \\
\hline 55 & 0.030 & 31 & 0.042 & 0.181 & 0.31 & 2.013 & 1.51 & 41.50 & $\mathrm{~F}$ \\
\hline 56 & 0.030 & 27 & 0.037 & 0.118 & 0.15 & 1.575 & 1.46 & 29.90 & $\mathrm{~S}$ \\
\hline 57 & 0.030 & 27 & 0.037 & 0.129 & 0.18 & 1.646 & 1.46 & 32.34 & $\mathrm{~S}$ \\
\hline 58 & 0.030 & 27 & 0.037 & 0.137 & 0.20 & 1.701 & 1.47 & 34.08 & $\mathrm{~S}$ \\
\hline 59 & 0.030 & 27 & 0.037 & 0.142 & 0.21 & 1.740 & 1.47 & 35.16 & $\mathrm{~S}$ \\
\hline 60 & 0.030 & 27 & 0.037 & 0.146 & 0.22 & 1.765 & 1.48 & 36.02 & F-S \\
\hline 61 & 0.030 & 27 & 0.037 & 0.150 & 0.23 & 1.788 & 1.47 & 36.87 & $\mathrm{~F}$ \\
\hline 62 & 0.030 & 27 & 0.037 & 0.157 & 0.23 & 1.695 & 1.37 & 38.35 & $\mathrm{~F}$ \\
\hline 63 & 0.030 & 27 & 0.037 & 0.170 & 0.27 & 1.792 & 1.39 & 41.06 & $\mathrm{~F}$ \\
\hline 64 & 0.030 & 27 & 0.042 & 0.140 & 0.20 & 1.701 & 1.45 & 34.73 & S \\
\hline 65 & 0.030 & 27 & 0.042 & 0.150 & 0.23 & 1.773 & 1.46 & 36.87 & S \\
\hline
\end{tabular}


Table 4. (Continued.)

\begin{tabular}{|c|c|c|c|c|c|c|c|c|c|}
\hline $\begin{array}{l}\text { Test } \\
\text { series }\end{array}$ & $\begin{array}{l}\text { Channel } \\
\text { slope, } S\end{array}$ & $\begin{array}{c}\text { Bank inclination, } \\
\alpha \text { (degrees) }\end{array}$ & $D_{50}(\mathrm{~m})$ & $h(\mathrm{~m})$ & $Q\left(\mathrm{~m}^{3} \mathrm{~s}^{-1}\right)$ & $V\left(\mathrm{~ms}^{-1}\right)$ & $F r$ & $\tau(\mathrm{Pa})$ & $\begin{array}{l}\text { Failed or } \\
\text { stable }\end{array}$ \\
\hline 66 & 0.030 & 27 & 0.042 & 0.150 & 0.23 & 1.780 & 1.47 & 36.87 & $\mathrm{~S}$ \\
\hline 67 & 0.030 & 27 & 0.042 & 0.155 & 0.24 & 1.811 & 1.47 & 37.93 & F-S \\
\hline 68 & 0.030 & 27 & 0.042 & 0.165 & 0.27 & 1.871 & 1.47 & 40.02 & $\mathrm{~F}$ \\
\hline 69 & 0.030 & 27 & 0.042 & 0.165 & 0.27 & 1.878 & 1.48 & 40.02 & $\mathrm{~F}$ \\
\hline 70 & 0.030 & 27 & 0.042 & 0.185 & 0.33 & 1.985 & 1.47 & 44.13 & $\mathrm{~F}$ \\
\hline 71 & 0.030 & 27 & 0.042 & 0.185 & 0.33 & 1.985 & 1.47 & 44.13 & $\mathrm{~F}$ \\
\hline 72 & 0.030 & 27 & 0.042 & 0.202 & 0.38 & 2.075 & 1.47 & 47.54 & $\mathrm{~F}$ \\
\hline 73 & 0.030 & 27 & 0.042 & 0.206 & 0.39 & 2.090 & 1.47 & 48.34 & $\mathrm{~F}$ \\
\hline 74 & 0.030 & 27 & 0.042 & 0.208 & 0.40 & 2.107 & 1.48 & 48.73 & $\mathrm{~F}$ \\
\hline 75 & 0.030 & 27 & 0.042 & 0.209 & 0.40 & 2.105 & 1.47 & 48.93 & $\mathrm{~F}$ \\
\hline 76 & 0.030 & 27 & 0.042 & 0.211 & 0.41 & 2.107 & 1.47 & 49.32 & $\mathrm{~F}$ \\
\hline 77 & 0.030 & 27 & 0.042 & 0.211 & 0.41 & 2.133 & 1.48 & 49.32 & $\mathrm{~F}$ \\
\hline 78 & 0.030 & 27 & 0.042 & 0.180 & 0.42 & 2.670 & 2.01 & 43.11 & $\mathrm{~F}$ \\
\hline 79 & 0.030 & 27 & 0.042 & 0.217 & 0.43 & 2.161 & 1.48 & 50.50 & $\mathrm{~F}$ \\
\hline 80 & 0.030 & 27 & 0.042 & 0.223 & 0.45 & 2.162 & 1.46 & 51.67 & $\mathrm{~F}$ \\
\hline 81 & 0.055 & 35 & 0.047 & 0.122 & 0.23 & 2.395 & 2.19 & 53.37 & $\mathrm{~S}$ \\
\hline 82 & 0.055 & 35 & 0.047 & 0.132 & 0.26 & 2.432 & 2.14 & 56.96 & $\mathrm{~S}$ \\
\hline 83 & 0.055 & 35 & 0.047 & 0.145 & 0.27 & 2.317 & 1.94 & 61.51 & F-S \\
\hline 84 & 0.055 & 35 & 0.047 & 0.148 & 0.28 & 2.331 & 1.94 & 62.55 & $\mathrm{~F}$ \\
\hline 85 & 0.055 & 35 & 0.047 & 0.164 & 0.30 & 2.209 & 1.74 & 67.94 & $\mathrm{~F}$ \\
\hline 86 & 0.055 & 35 & 0.047 & 0.175 & 0.31 & 2.133 & 1.63 & 71.56 & $\mathrm{~F}$ \\
\hline 87 & 0.055 & 35 & 0.037 & 0.095 & 0.14 & 1.851 & 1.92 & 43.21 & S \\
\hline 88 & 0.055 & 35 & 0.037 & 0.099 & 0.15 & 1.900 & 1.93 & 44.76 & S \\
\hline 89 & 0.055 & 35 & 0.037 & 0.100 & 0.16 & 2.022 & 2.04 & 45.14 & $\mathrm{~S}$ \\
\hline 90 & 0.055 & 35 & 0.037 & 0.109 & 0.18 & 2.123 & 2.05 & 48.56 & F-S \\
\hline 91 & 0.055 & 35 & 0.037 & 0.113 & 0.20 & 2.267 & 2.15 & 50.06 & $\mathrm{~F}$ \\
\hline 92 & 0.055 & 35 & 0.037 & 0.118 & 0.22 & 2.323 & 2.16 & 51.90 & $\mathrm{~F}$ \\
\hline 93 & 0.055 & 35 & 0.042 & 0.115 & 0.23 & 2.557 & 2.41 & 50.80 & S \\
\hline 94 & 0.055 & 35 & 0.042 & 0.119 & 0.24 & 2.569 & 2.38 & 52.27 & $\mathrm{~S}$ \\
\hline 95 & 0.055 & 35 & 0.042 & 0.123 & 0.25 & 2.580 & 2.35 & 53.73 & F-S \\
\hline 96 & 0.055 & 35 & 0.042 & 0.139 & 0.27 & 2.385 & 2.04 & 59.43 & $\mathrm{~F}$ \\
\hline 97 & 0.055 & 35 & 0.042 & 0.145 & 0.29 & 2.446 & 2.05 & 61.51 & $\mathrm{~F}$ \\
\hline 98 & 0.055 & 35 & 0.042 & 0.153 & 0.30 & 2.455 & 2.01 & 64.25 & $\mathrm{~F}$ \\
\hline
\end{tabular}

Note: $\mathrm{F}=$ failed test; $\mathrm{S}=$ stable test; and F-S = transitional test.

Table 5. Test program with two layers of blocks with $D_{50}=0.037 \mathrm{~m}$

\begin{tabular}{|c|c|c|c|c|c|c|c|c|}
\hline Test series & $\begin{array}{c}\text { Channel } \\
\text { slope } \\
(\%)\end{array}$ & $\begin{array}{c}\text { Bank } \\
\text { inclination, } \\
\alpha \text { (degrees) }\end{array}$ & $Q\left(\mathrm{~m}^{3} \mathrm{~s}^{-1}\right)$ & $h(\mathrm{~m})$ & $V\left(\mathrm{~ms}^{-1}\right)$ & $F r$ & $\tau(\mathrm{Pa})$ & $\begin{array}{c}\text { Failed or } \\
\text { stable }\end{array}$ \\
\hline Double 1 & 0.015 & 35 & 0.340 & 0.233 & 1.684 & 1.11 & 24.40 & S \\
\hline Double 2 & 0.015 & 35 & 0.347 & 0.235 & 1.701 & 1.12 & 24.56 & F-S \\
\hline Double 3 & 0.015 & 35 & 0.355 & 0.239 & 1.706 & 1.11 & 24.88 & $\mathrm{~F}$ \\
\hline Double 4 & 0.015 & 35 & 0.380 & 0.248 & 1.747 & 1.12 & 25.59 & $\mathrm{~F}$ \\
\hline Double 5 & 0.030 & 35 & 0.310 & 0.150 & 1.867 & 1.54 & 36.66 & S \\
\hline Double 6 & 0.030 & 35 & 0.333 & 0.163 & 1.830 & 1.45 & 39.32 & F-S \\
\hline Double 7 & 0.030 & 35 & 0.350 & 0.168 & 1.860 & 1.45 & 40.33 & $\mathrm{~F}$ \\
\hline Double 8 & 0.030 & 35 & 0.375 & 0.182 & 1.823 & 1.37 & 43.10 & $\mathrm{~F}$ \\
\hline Double 9 & 0.030 & 31 & 0.188 & 0.132 & 1.758 & 1.55 & 31.92 & $\mathrm{~S}$ \\
\hline Double 10 & 0.030 & 31 & 0.200 & 0.140 & 1.749 & 1.49 & 33.54 & F-S \\
\hline Double 11 & 0.030 & 31 & 0.228 & 0.151 & 1.842 & 1.52 & 35.53 & $\mathrm{~F}$ \\
\hline Double 12 & 0.030 & 31 & 0.255 & 0.167 & 1.820 & 1.42 & 38.84 & $\mathrm{~F}$ \\
\hline Double 13 & 0.030 & 27 & 0.218 & 0.145 & 1.779 & 1.49 & 35.80 & S \\
\hline Double 14 & 0.030 & 27 & 0.228 & 0.157 & 1.695 & 1.37 & 38.35 & F-S \\
\hline Double 15 & 0.030 & 27 & 0.240 & 0.162 & 1.719 & 1.36 & 39.40 & $\mathrm{~F}$ \\
\hline Double 16 & 0.030 & 27 & 0.265 & 0.170 & 1.792 & 1.39 & 41.06 & $\mathrm{~F}$ \\
\hline Double 17 & 0.055 & 35 & 0.180 & 0.109 & 2.123 & 2.05 & 48.56 & S \\
\hline Double 18 & 0.055 & 35 & 0.200 & 0.117 & 2.182 & 2.04 & 51.54 & F-S \\
\hline Double 19 & 0.055 & 35 & 0.215 & 0.122 & 2.239 & 2.05 & 53.37 & $\mathrm{~F}$ \\
\hline Double 20 & 0.055 & 35 & 0.230 & 0.127 & 2.290 & 2.05 & 55.17 & $\mathrm{~F}$ \\
\hline Double 21 & 0.055 & 35 & 0.245 & 0.138 & 2.223 & 1.91 & 59.08 & $\mathrm{~F}$ \\
\hline
\end{tabular}

$\mathrm{F}=$ failed test $\mathrm{S}=$ stable test; and $\mathrm{F}-\mathrm{S}=$ transitional test. 
reach of the flume between 6.5 and $9.5 \mathrm{~m}$ where a constant flow depth with roughly uniform flow occurred. Erosion occurring downstream of the observation zone was excluded by fixing the blocks.

Twenty-one additional tests were conducted to analyze the effect of a second layer on the stability of packed riprap [Fig. 5(a)] Tests with two layers were also run for a maximum of $180 \mathrm{~min}$ until total failure of the riprap occurred in a certain cross section. Fig. 5(b) shows the riprap before and after the test with two layers (test Double 8 according to Table 5).

Herein, the unit discharge $q$ is calculated from total discharge by dividing it by the top channel width of the trapezoidal section. The mean flow velocity $V$ is obtained from total discharge $Q$ divided by the wetted area $A$. The shear stress is defined as $\tau=\rho g h S$, where $\rho$ is water density $\left(\mathrm{kgm}^{-3}\right), g$ is acceleration of gravity $\left(\mathrm{ms}^{-2}\right), h$ is flow depth (m), and $S$ is channel slope.

The accuracy of the data is related to the error in the measurements of discharge, which is $0.5 \%$, and the measurements of flow depth, which is $0.5 \%$.

\section{Results and Discussion}

\section{Comparison of the Experimental Results with Riprap Sizing Equations from the Literature}

Some of the tests with dumped blocks of $D_{50}=0.037$ and $0.042 \mathrm{~m}$ for a channel slope of 3\% and bank inclination of $\alpha=35^{\circ}$ are compared with the equations developed by EM-1601 [Eq. (4)] and HEC-11 [Eq. (5)]. In Fig. 6, the experimental results are presented in terms of relative roughness $D / h$ as a function of the modified Froude number

$$
F r^{*}=\frac{V}{\sqrt{\left(S_{B}-1\right) g h}}
$$

where $V=$ mean flow velocity; $S_{B}=$ specific gravity of the blocks; $h=$ flow depth; and $g=$ gravitational acceleration, where a distinction is made between stable and failed tests.

Furthermore, in Fig. 6, the equations of HEC-11 and EM-1601 are presented for $\alpha=35^{\circ}$

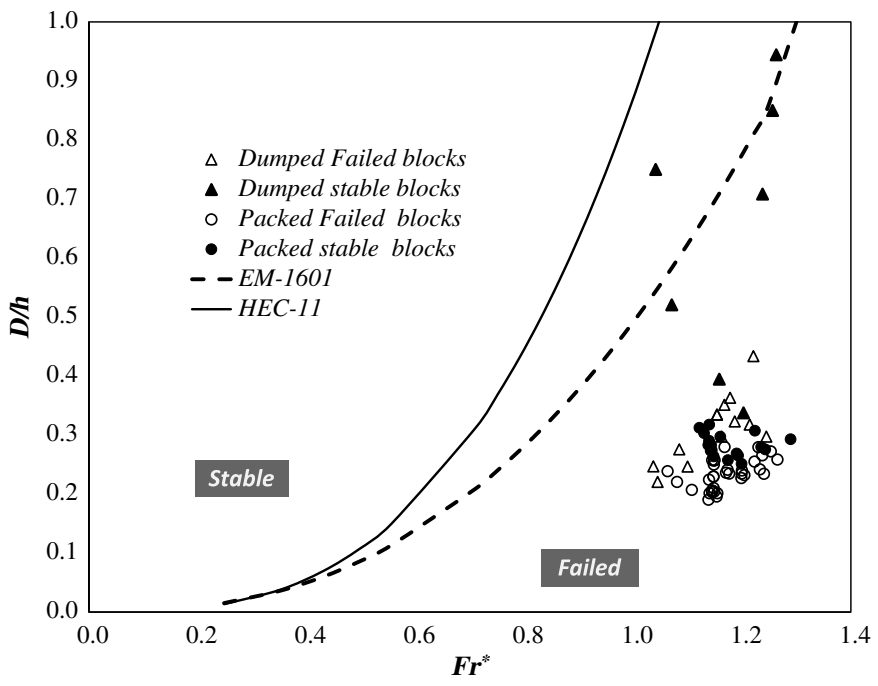

Fig. 6. Relative roughness $D / h$ as a function of $F r^{*}=V /$ $\left[\left(S_{B}-1\right) g h\right]^{0.5}$ for dumped and packed riprap with a bank inclination of $35^{\circ}$ compared with equations of HEC-11 and EM-1601.

$$
\frac{D_{30}}{h}=0.30 C\left(K_{1}\right)\left[\frac{V}{\sqrt{\left(S_{B}-1\right) g h}}\right]^{2.5}
$$

The equation of HEC-11 becomes

$$
\frac{D_{50}}{h}=0.295 C\left(K_{2}\right)\left[\frac{V}{\sqrt{\left(S_{B}-1\right) g h}}\right]^{3}
$$

where $C\left(K_{1}\right)$ and $C\left(K_{2}\right)=$ coefficients of the bank inclination (Lagasse et al. 2006).

To see the differences in behavior of dumped and packed riprap, the results of 32 experiments with packed riprap, including failed and stable tests, having the same block sizes but higher velocities (Table 4) have been added in Fig. 6. A significant shift in the location of the stable tests of dumped riprap can be observed in Fig. 6 . The tests with packed riprap clearly indicate that the existing design equations would overestimate the required block size. The reason is that the failure mechanisms of dumped and packed riprap are different. Some limited erosion of the packed blocks was observed even if the section remained stable. From this point of view, the equations could be valid only for the dumped median size of the blocks on the plain bed.

\section{Block Erosion Rate for Packed and Dumped Riprap}

In all experiments, the erosion rate was measured by counting the number of eroded blocks per minute based on a video analysis and converting this information into kilograms per second using the unit weight of the blocks. The block erosion transport rate $q_{s}$ as a function of the unit water discharge $q$ is shown in Fig. 7 for dumped and packed riprap tests on a channel slope of $3 \%$. For each tested water depth, the unit discharge $q$ is calculated based on the top channel width for the half-trapezoidal section.

For dumped riprap, the start of block erosion was close to complete failure because total bank failure always occurs rapidly, given that the blocks are less supported by each other. The packed riprap remains stable for a longer time. The discharge that initiates block movement is slightly higher for packed riprap. The erosion rate increases with increasing unit discharge. However, due to the interlocking forces among the blocks, less block erosion was observed in packed riprap.

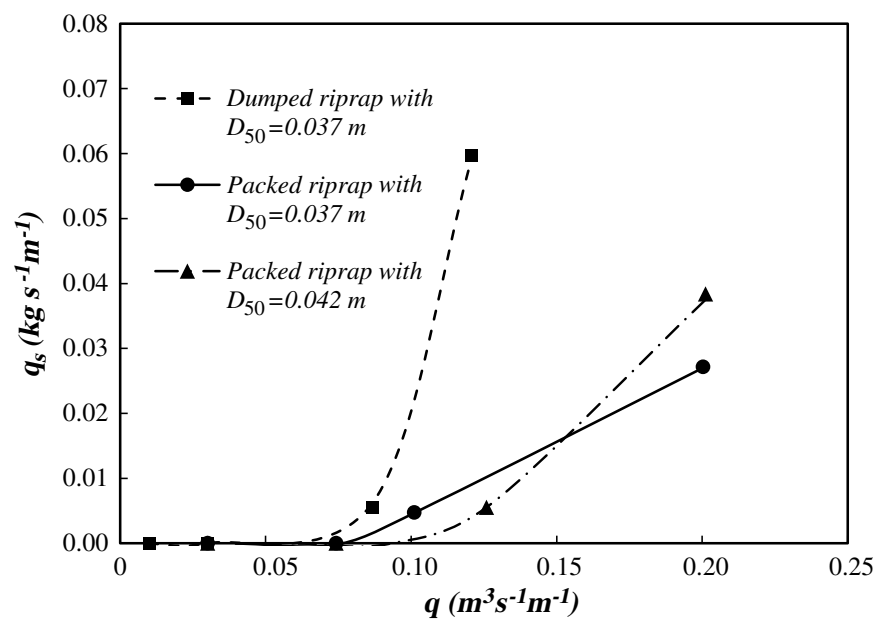

Fig. 7. Block erosion rate $q_{s}\left(\mathrm{kgs}^{-1} \mathrm{~m}^{-1}\right)$ as a function of the unit discharge $q\left(\mathrm{~m}^{3} \mathrm{~s}^{-1} \mathrm{~m}^{-1}\right)$ for a block size of $D_{50}=0.037 \mathrm{~m}$ (dumped and packed) and $D_{50}=0.042 \mathrm{~m}$ (packed). 


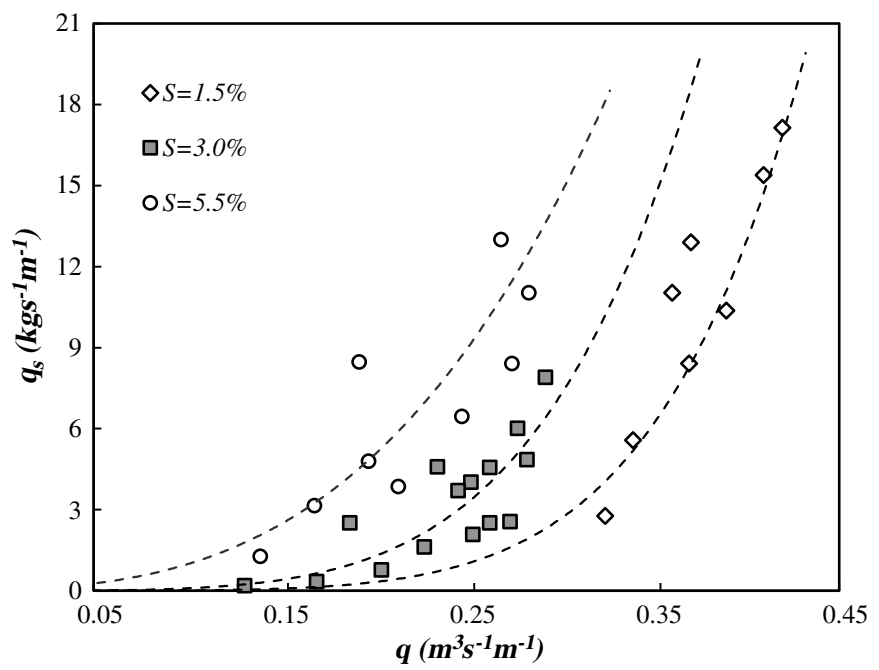

Fig. 8. Block erosion rate $q_{s}\left(\mathrm{kgs}^{-1} \mathrm{~m}^{-1}\right)$ as a function of the unit discharge $q\left(\mathrm{~m}^{3} \mathrm{~s}^{-1} \mathrm{~m}^{-1}\right)$ for different channel slopes.

To highlight the effect of the channel slope, the block erosion transport rate was measured for 32 packed riprap tests that remained stable during the first hour of testing. These tests include three block sizes for three different channel slopes and a bank inclination of $35^{\circ}$ (Table 4 ). The influence of the longitudinal channel slope on the block erosion transport rate $q_{s}\left(\mathrm{kgs}^{-1} \mathrm{~m}^{-1}\right)$ can be seen in Fig. 8. As expected, for a given unit discharge $(q)$, a higher block erosion rate occurs for steeper slopes.

For each tested channel slope, an exponential increase in the block erosion rate with the unit discharge was observed. This is also the case if the block erosion rate is presented as a function of the dimensionless bed shear stress, $\theta=0.77 \tau /\left(S_{B}-1\right) D_{B}$, as shown in Fig. 9, where $\tau$ is the shear stress, $S_{B}$ is the specific gravity of the blocks, and $D_{B}$ is the diameter of the blocks. The factor of 0.77 reflects the distribution of the bed shear stress across the section, which is smaller at the toe of the riprap than in the center of the section (Chow 1959).

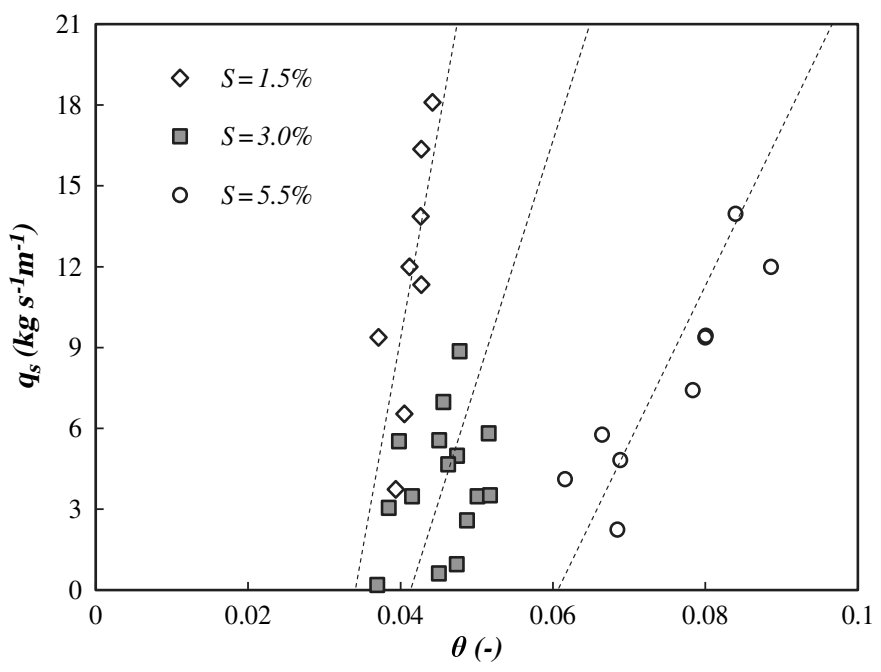

Fig. 9. Block erosion rate $q_{s}\left(\mathrm{kgs}^{-1} \mathrm{~m}^{-1}\right)$ as a function of the dimensionless shear stress $\theta=0.77 \tau /\left(S_{B}-1\right) D_{B}$ for a riprap with a bank inclination of $35^{\circ}$. Experimental data are grouped per channel slope.
The block size does not influence the block erosion rate, whereas the channel slope has a significant influence. The block erosion rate tends to increase linearly with the dimensionless bed shear stress $\theta$. This block erosion rate linearly depends on the channel slope (Fig. 9).

\section{Design of the Riprap with Packed, Individually Placed Large Blocks}

\section{Design Equation for the Required Block Size}

The results of all 98 performed tests with packed riprap are evaluated in terms of nondimensional block size $D / h$ (i.e., relative roughness) and the modified Froude number. In Fig. 10, the results are grouped into three categories named failed tests, stable tests, and transitional tests. In addition to the stable and failed tests, to define the boundary between these tests, transitional tests were also performed (Table 4). The failed and transitional tests are characterized by the lowest discharge causing a failure. As indicated in Fig. 10, the failed, stable, and transitional tests are grouped according to the different channel slopes.

Given that the relative block size $D / h$ (i.e., relative roughness) is zero when the velocity (i.e., modified Froude number) is zero, the transitional tests (i.e., limit between the stable and failed tests) can be expressed as follows $\left(R^{2}=0.93\right)$ :

$$
\frac{D}{h} \geq 0.22\left(\frac{V}{\sqrt{\left(\left(S_{B}-1\right) g h\right)}}\right)^{0.88}
$$

Eq. (6) can be used for the estimation of the required block size in the case of packed riprap if supercritical flow conditions are prevailing, and in the following range of application of modified Froude numbers $0.6<F r * \leq 2$, the relative roughness is $0.1<$ $D / h \leq 0.4$, and the slopes are $1.5 \% \leq S \leq 5.5 \%$. Furthermore, the relationship is valid for a bank inclination of $27 \leq \leq 35 \alpha$. If a scale of 1:25 is considered, the ranges of block sizes in the prototype are $D_{B}=0.91 \mathrm{~m} \pm 9.6 \%$ (Class I), $D_{B}=1.08 \mathrm{~m} \pm 4.7 \%$

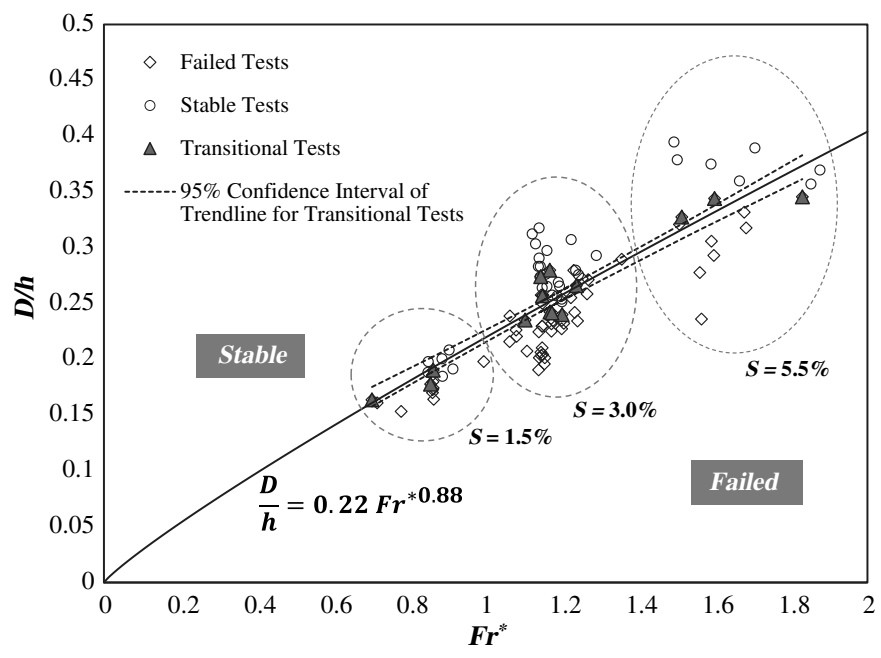

Fig. 10. Relative roughness $D / h$ (or nondimensional block size) as a function of $\mathrm{Fr}^{*}=V /\left[\left(S_{B}-1\right) g h\right]^{0.5}$. Comparison of the failed, stable, and transitional tests for channel slopes of $1.5 \%, 3 \%$, and $5.5 \%$ and riprap bank inclinations of $35^{\circ}, 31^{\circ}$, and $27^{\circ}$ (Table 4 ). The regression (solid line) corresponds to the trendline of the transitional tests, and the dashed lines indicate a $95 \%$ probability of a true best fit. 
Table 6. Correction factor considering the bank inclination [Eq. (8)] for packed riprap; comparison with the correction factor of the design formulas for dumped riprap

\begin{tabular}{lcccccc}
\hline $\begin{array}{l}\text { Bank } \\
\text { inclination, } \\
\alpha \text { (degrees) }\end{array}$ & $C$ & $C_{f}$ & $R^{2}$ & Exponent & $\begin{array}{c}C(K 1) \\
(\text { EM-1601) }\end{array}$ & $\begin{array}{c}C(K 2) \\
(\text { HEC-11) }\end{array}$ \\
\hline 40 & 0.236 & 1.07 & - & 0.89 & 0.30 & 0.296 \\
35 & 0.228 & 1.04 & 0.94 & 0.88 & 0.28 & 0.2601 \\
31 & 0.217 & 0.99 & 0.91 & 0.88 & 0.26 & 0.2322 \\
27 & 0.215 & 0.97 & 0.97 & 0.88 & 0.23 & 0.2022 \\
\hline
\end{tabular}

(Class II), and $D_{B}=1.2 \mathrm{~m} \pm 4.2 \%$ (Class III). The block weight range is then varied as $W_{\text {ClassI }}=1,057 \mathrm{~kg} \pm 28 \%, W_{\text {ClassII }}=$ $1,729 \mathrm{~kg} \pm 14 \%$, and $W_{\text {ClassIII }}=2,405 \mathrm{~kg} \pm 12 \%$ in the prototype.

\section{Influence of the Bank Inclination}

The bank inclination influences the stability of the riprap. To define a correction factor $C_{f}$ that reflects the influence of the bank inclination for the riprap stability, as indicated in Eq. (7), the experimental results have been analyzed for the tested riprap bank inclination assuming an angle of repose of $\varphi\left(=40^{\circ}\right)$ for the blocks

$$
D_{50} \geq 0.22 C_{f} h\left(\frac{V}{\sqrt{\left(\left(S_{B}-1\right) g h\right)}}\right)^{0.88}
$$

The best fit $\left(R^{2}=0.94\right)$ with the experimental data was obtained with a bank-inclination correction factor $C_{f}$ defined as follows:

$$
C_{f}=1.073\left[\frac{\tan (\alpha)}{\tan (\varphi)}\right]^{0.2}
$$

The preceding bank-inclination correction factors $C_{f}$ are compared with the coefficients of Maynord and Neil's (2008) equations (EM-1601) and HEC-11 as $C\left(K_{1}\right)$ and $C\left(K_{2}\right)$, respectively, in Table 6. The exponent of the modified Froude number is given in Table 7. By increasing the bank inclination, $C_{f}$ increases, and consequently, the required block sizes have to be larger in order to resist erosion. For riprap with large, individually placed blocks in relatively steep mountain rivers, the required block size can be obtained by Eq. (7) using the bank-inclination correction factor according to Eq. (8). For the design of the required block size in Eq. (7), normally, a safety factor applied to the discharge of the design flood is recommended, which depends on the mean flow velocity and flow depth. Thus, this safety factor is not explicated and included in Eq. (7).

\section{Influence of a Second Riprap Layer}

The relationship between the relative roughness $D / h$ and modified Froude number $\left(\mathrm{Fr}^{*}\right)$ is shown in Fig. 11 separately for single- and double-layer riprap. The stable and failed tests are illustrated by individual symbols for the single and double layers. The transitional tests define the boundary between failure and stable conditions. For $0.8<F r^{*} \leq 1.8$, the transitional tests for the double-layer riprap can be fitted in good agreement with Eq. (9) $\left(R^{2}=88\right)$. For a given block size, a second layer results in an increased stability of the riprap

$$
\frac{D}{h} \geq 0.205\left(\frac{V}{\sqrt{\left(\left(S_{B}-1\right) g h\right)}}\right)^{1.12}
$$

\section{Design Example}

In this section, the application of Eq. (7) to riprap consisting of individually placed blocks as an example of a typical alpine river is illustrated and compared with other approaches. An alpine river with the following characteristics is considered: bed width $b$ of $15 \mathrm{~m}$, bed slope $S$ of $4 \%$, bank inclination of $34^{\circ}$, and Manning roughness coefficient of the river section $n=0.04 \mathrm{~m}^{1 / 3} \mathrm{~s}^{-1}$. The large blocks have a specific gravity of $S_{B}=2.65$. For blocks heavier than $1 \mathrm{t}$, according Froehlich (2013) an angle of repose of $\varphi=45^{\circ}$ can be assumed. In Table 7 , the required block size for the limit state of stability [safety factor $(\mathrm{SF})=1$ ] at the bank toe is given for varying discharges corresponding to different water depths estimated with the Manning-Strickler equation and assuming uniform flow conditions.

The placed riprap should resist to a flood of $H Q_{100}=$ $600 \mathrm{~m}^{3} \mathrm{~s}^{-1}$ with $\mathrm{SF}=1.3$. Thus, the riprap should be still at its limit state of stability for a discharge of $Q=1.3 \times H Q_{100}=$ $780 \mathrm{~m}^{3} \mathrm{~s}^{-1}$. For riprap composed by individually placed blocks, Eq. (7) results in a required block diameter of $D_{B 50}=1.07 \mathrm{~m}$, resulting in a weight of approximately $1.7 \mathrm{t}$. The approach of EM-1601 developed for dumped riprap would require an unreasonable large block diameter of $D_{B 30}=2.67 \mathrm{~m}$ (i.e., a block weight approximately $26 \mathrm{t}$ ). According to the experience reported by Schleiss (2000), the method of Stevens et al. (1976) can be applied reasonably in steep mountain rivers if a critical dimensionless shear stress $\theta_{c r}=0.1$ is used instead of 0.047 . For the design example with $Q=780 \mathrm{~m}^{3} \mathrm{~s}^{-1}$, this would result in a required block diameter of $D_{m}=1.19 \mathrm{~m}$ with a weight of about $2.3 \mathrm{t}$, which is significantly heavier than the required block according to Eq. (7) of $1.7 \mathrm{t}$.

If the riprap is designed with two layers of the same block diameter of $D_{B 50}=1.07 \mathrm{~m}$, according to Fig. 11, it would resist erosion at a flow depth of $4.87 \mathrm{~m}$ instead of $4.52 \mathrm{~m}$, which corresponds to a flood discharge of $Q=860 \mathrm{~m}^{3} \mathrm{~s}^{-1}$. The second layer may therefore give significant additional safety. Jafarnejad et al. (2017a) also analyzed the time to failure of riprap with individually placed large blocks, where failure was defined as the total collapse of all blocks in a section over the whole bank; thus, the failure mechanism considered herein is sliding. The method for estimating the time to failure provided by Jafarnejad et al. (2017a) was applied to this example. A time to failure of $65 \mathrm{~h}$ before complete failure of the

\begin{tabular}{|c|c|c|c|c|c|c|c|}
\hline $\begin{array}{l}\mathrm{HQ} \\
\left(\mathrm{m}^{3} \mathrm{~s}^{-1}\right)\end{array}$ & $\begin{array}{c}\text { Flow depth, } \\
h(\mathrm{~m})\end{array}$ & $\begin{array}{c}V \\
\left(\mathrm{~ms}^{-} 1\right)\end{array}$ & $\begin{array}{c}D_{B 30}(\mathrm{~m}) \\
(\mathrm{EM}-1601)\end{array}$ & $\begin{array}{l}D_{B m}(\text { Stevens } \\
\text { et al. 1976) (m) }\end{array}$ & $\begin{array}{c}D_{B 50} \text { Authors' } \\
\text { Eq. (7) (m) }\end{array}$ & $\begin{array}{c}t_{f} \text { one } \\
\text { layer }(\mathrm{h})\end{array}$ & $\begin{array}{c}t_{f} \text { two } \\
\text { layers }(\mathrm{h})\end{array}$ \\
\hline 200 & 2.04 & 5.93 & 1.03 & 0.61 & 0.46 & 16 & 37 \\
\hline 400 & 3.07 & 7.54 & 1.70 & 0.92 & 0.71 & 55 & 74 \\
\hline 600 & 3.89 & 8.63 & 2.25 & 1.18 & 0.91 & 86 & 100 \\
\hline 780 & 4.52 & 9.38 & 2.67 & 1.37 & 1.07 & 109 & 120 \\
\hline 900 & 4.91 & 9.82 & 2.93 & 1.49 & 1.16 & 124 & 131 \\
\hline
\end{tabular}
riprap can be estimated for the flood peak of the design discharge of

Table 7. Riprap design example for a typical alpine river

Note: Bold value indicates the design flood and block diameters for a safety factor of 1.3. 


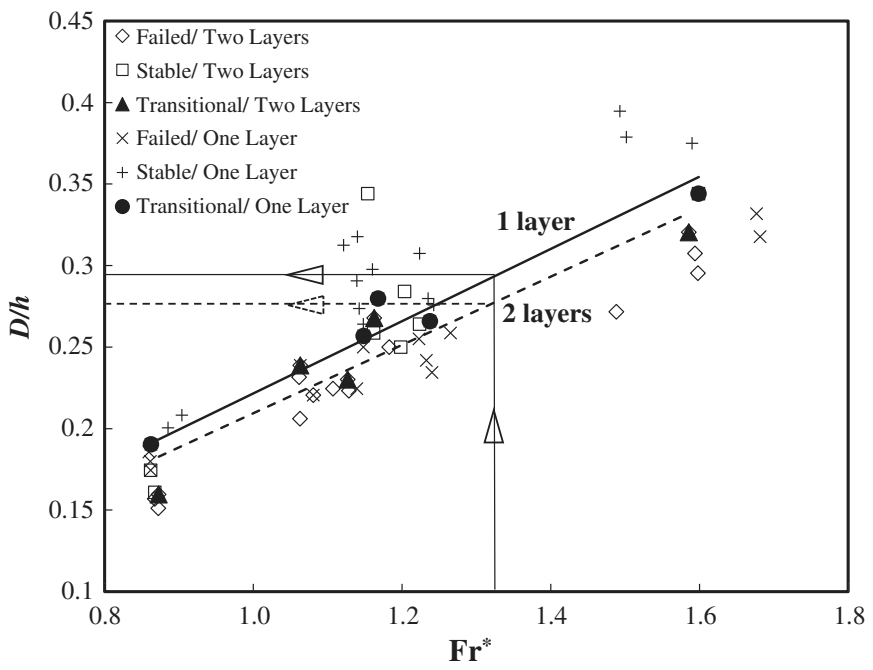

Fig. 11. Relative roughness $(D / h)$ as a function of $F r *$ for the stable, critical, and failed tests for one (straight line) and two layers (dashed line) of riprap.

$Q=780 \mathrm{~m}^{3} \mathrm{~s}^{-1}$. A second layer of riprap would delay complete failure from 109 to $120 \mathrm{~h}$.

\section{Conclusions}

If riprap is used as bank protection in mountain rivers, then heavy and large blocks (large extracted rocks from quarries) are typically required and have to be placed individually by mechanical methods. The stability of such packed riprap was experimentally investigated with 98 flume tests carried out for supercritical flow conditions with varying flume slopes, bank inclinations, and block sizes. An additional 34 tests were performed for dumped riprap, and a comparison with existing riprap design methods was performed. Furthermore, the effect of a second layer on the stability of packed riprap was assessed with 21 tests. The influence of the channel bank inclination was assessed by means of a correction factor.

For the experimental results using dumped riprap, a good agreement with the HEC-11 and EM-1601 design equations was obtained, particularly when considering the bank-inclination effect and the boundary between stable or failed conditions. However, the design equations overestimated the required block size in some of the tests performed with dumped riprap.

Compared with dumped riprap, the tests performed with packed riprap revealed a clearly higher stability with a delay in failure and a smaller block erosion rate after failure. The channel slope had the strongest effect on the block erosion rate. Furthermore, the experiments showed that block erosion decreased significantly for packed riprap due to the better interlocking forces between the blocks.

For the tested range of application (defined here with slope and other nondimensional parameters), an improved design equation [Eq. (7)] for packed riprap was proposed, resulting in the required nondimensional block size (i.e., relative roughness) as a function of the modified Froude number [Eq. (3)]. Such a packed riprap, where large blocks are placed individually by machines, had a significantly higher resistance against erosion than dumped riprap when using the same large block size. Dumped riprap failed more rapidly once the first block was eroded.
The application range of the improved design equations is limited by Froude numbers $F r$ of 0.90 and 2.41, channel slopes $S$ from 0.015 to 0.055 , and angle bank inclinations between $27^{\circ}$ and $35^{\circ}$. The tests were performed with blocks having a specific gravity of 2.66 and an angle of repose between $40^{\circ}$ and $41^{\circ}$. Caution should be used when applying the equations outside the bounds under which they were developed.

The experiments revealed that, for the same longitudinal channel slope and bank inclination, a second layer of packed riprap stabilized the section and postponed failure. However, when failure started, the block erosion rate was increased significantly by the second layer. For higher discharges that caused failure, the effect of the second layer on stabilizing the section was less pronounced. This means that for low discharges, the thickness (i.e., second layer of the riprap) has more influence on stability. Regarding the bank inclination, a double layer makes the riprap more stable for higher inclinations compared with the single-layer riprap. The second layer had a greater stabilizing role when the riprap inclination approached the angle of repose of the blocks. Therefore, the same stability can be achieved with smaller blocks when placing them in a double layer compared with a single layer of riprap.

\section{Acknowledgments}

This research was funded by the Swiss Federal Office for the Environment (FOEN) under Contract No. A2111.0239/10-Q8 0019.PJ/J372-1192.

\section{Notation}

The following symbols are used in this paper:

$C_{f}=$ bank-inclination correction factor;

$C\left(K_{1}\right)=$ bank-inclination correction factor of EM-1601;

$C\left(K_{2}\right)$ = bank-inclination correction factor of HEC-11;

$D=$ characteristic particle diameter $(\mathrm{m})$;

$D_{B}=$ characteristic block diameter $(\mathrm{m})$;

$F r=$ Froude number;

$\mathrm{Fr}^{*}=$ modified Froude number;

$g=$ acceleration of gravity $\left(\mathrm{ms}^{-2}\right) ;$

$\mathrm{HQ}=$ design discharge $\left(\mathrm{m}^{3} \mathrm{~s}^{-1}\right)$;

$h=$ flow depth (m);

$L=$ length (m);

$Q=$ total discharge $\left(\mathrm{m}^{3} \mathrm{~s}^{-1}\right)$;

$q=$ unit discharge $\left(\mathrm{m}^{3} \mathrm{~s}^{-1} \mathrm{~m}^{-1}\right)$;

$q_{s}=$ block erosion transport rate $\left(\mathrm{kgs}^{-1} \mathrm{~m}^{-1}\right)$;

$S=$ channel slope;

$S_{B}=$ specific block gravity $=\rho_{s} / \rho$;

$T=$ time (s);

$t_{f}=$ time to failure $(\mathrm{h})$;

$u^{*}=$ friction velocity $\left(\mathrm{ms}^{-1}\right)$;

$V=$ mean flow velocity $\left(\mathrm{ms}^{-1}\right)$;

$V_{c}=$ characteristic flow velocity $\left(\mathrm{ms}^{-1}\right)$;

$\alpha=$ bank inclination (degrees);

$\theta=$ dimensionless bed shear stress;

$\lambda_{T}=$ time scale (s);

$\rho=$ water density $\left(\mathrm{kgm}^{-3}\right)$;

$\rho_{s}=$ particle density $\left(\mathrm{kgm}^{-3}\right)$;

$\tau=$ shear stress $(\mathrm{Pa})$;

$\theta_{c r}=$ critical dimensionless bed shear stress; and

$\varphi=$ angle of repose of the blocks (degrees). 


\section{References}

Abt, S. R., and T. L. Johnson. 1991. "Riprap design for overtopping flow." J. Hydraul. Eng. 117 (8): 959-972. https://doi.org/10.1061/(ASCE) 0733-9429(1991)117:8(959).

Abt, S. R., C. I. Thornton, H. A. Gallegos, and C. M. Ullmann. 2008. "Round-shaped riprap stabilization in overtopping flow." J. Hydraul. Eng. 134 (8): 1035. https://doi.org/10.1061/(ASCE)0733-9429(2008) 134:8(1035).

Ahmed, A. F. 1987. "Stability of riprap side slopes in open channels." Ph.D. thesis, Dept. of Civil Engineering, Univ. of Southampton.

Anderson, A. G., A. S. Paintal, and J. T. Davenport. 1970. Tentative design procedure for riprap lined channels. National Cooperative Highway Research Program Rep. No. 108. Minneapolis: Univ. of Minnesota.

Bezzola, G. R., and C. Hegg, eds. 2007. Ereignisanalyse Hochwasser 2005, Teil 1-Prozesse, Schäden und erste Einordnung. Bern, Switzerland: Bundesamt für Umwelt BAFU.

Bezzola, G. R., and C. Hegg, eds. 2008. Ereignisanalyse Hochwasser 2005, Teil 2-Analyse von Prozessen, Massnahmen und Gefahrengrundlagen. Bern, Switzerland: Bundesamt für Umwelt BAFU.

Blodgett, J. C., and C. E. McConaughy. 1986. Rock riprap design for protection of stream channels near highway structures; Volume 2, Evaluation of riprap design procedures. Water-Resources Investigations Rep. No. 86-4128. Sacramento, CA: USGS.

Brown, S. A., and E. S. Clyde. 1989. Design of riprap revetment. Hydraulic Engineering Circular 11. McLean, VA: Federal Highway Administration.

Chow, V. T. 1959. Open channel hydraulics. New York: McGraw-Hill.

De Almeida, G. A. M., and J. P. Martin-Vide. 2009. "Riprap stability: Transverse and longitudinal versus continues protections." J. Hydraul. Eng. 135 (6): 447-456. https://doi.org/10.1061/(ASCE)HY.1943-7900 .0000031 .

Escarameia, M., and R. W. P. May. 1992. Channel protection. Turbulence downstream of structures. Rep. No. SR313. London: HR Wallingford.

Froehlich, D. C. 2013. "Sizing loose rock riprap to protect stream bank." River Res. Appl. 29 (2): 219-235. https://doi.org/10.1002/rra.1587.

Froehlich, D. C., and C. A. Benson. 1996. "Sizing dumped rock riprap." J. Hydraul. Eng. 122 (7): 389-396. https://doi.org/10.1061/(ASCE) 0733-9429(1996)122:7(389).

Grace, J. L., Jr., C. C. Calhoun, Jr., and D. N. Brown. 1973. Drainage and erosion control facilities: Field performance investigation. Miscellaneous Paper H-73-6. Vicksburg, MS: US Army Engineer Waterways Experiment Station.

Hiller, P. H., J. Aberle, and L. Lia. 2017. "Displacements as failure origin of placed riprap on steep slopes.” J. Hydraul. Res. 56 (2): 141-155. https:// doi.org/10.1080/00221686.2017.1323806.

Isbash, S. V. 1935. Construction of dams by dumping stones in flowing water. Eastport, ME: War Dept., US Engineer Office, Engineering Division.

Jafarnejad, M., M. J. Franca, M. Pfister, and A. J. Schleiss. 2017a. "Timebased failure analysis of compressed riverbank riprap." J. Hydraul. Res. 55 (2): 224-235. https://doi.org/10.1080/00221686.2016.1212940.

Jafarnejad, M., M. Pfister, E. Brühwiler, and A. J. Schleiss. 2017b. "Probabilistic failure analysis of riprap as riverbank protection under flood uncertainties." J. Stochastic Environ. Res. Risk Assess. 31 (7): 1839-1851. https://doi.org/10.1007/s00477-016-1368-6.

Lagasse, P. F., P. E. Clopper, L. W. Zevenbergen, and J. F. Ruff. 2006. Riprap design criteria, recommended specifications, and quality control. NCHRP Rep. No. 568. Washington, DC: Transportation Research Board.

Lane, E. W. 1952. Progress report on results of studies on design of stable channels. Hydraulic Laboratory Rep. No. HYD-352. Denver: Bureau of Reclamation.
Li, R. M., D. B. Simons, P. H. Blinco, and M. A. Samad. 1976. "Probabilistic approach to design of riprap riverbank protection." In Vol. 1 of Proc., Symp. on Inland Waterways for Navigation, Flood Control, and Water Diversions, 1572-1591. Reston, VA: ASCE.

Maynord, S. T. 1992. Riprap stability: Studies in near-prototype size laboratory channel. Technical Rep. No. HL-92-5. Vicksburg, MS: US Army Engineer Waterways Experiment Station.

Maynord, S. T., and C. Neill. 2008. "Riprap design.” In Sedimentation engineering: ASCE manual and reports on engineering practice no. 110, edited by M. H. García, 1037-1056. Reston, VA: ASCE.

Maynord, S. T., J. F. Ruff, and S. R. Abt. 1989. "Riprap design." J. Hydraul. Eng. 115 (7): 937-949. https://doi.org/10.1061/(ASCE)0733 -9429(1989)115:7(937).

Maynord, S. T., J. F. Ruff, and S. R. Abt. 1991. "Closure of discussion on riprap design.” J. Hydraul. Eng. 117 (4): 542-544. https://doi.org/10 .1061/(ASCE)0733-9429(1991)117:4(542.2).

Pilarczyk, K. W. 1990. "Coastal protection.” In Short course on coastal protection. Rotterdam, Netherlands: A.A. Balkema.

Racin, J. A., T. P. Hoover, and C. M. Crossett Avila. 2000. California bank and shore rock slope protection design: Practitioner's guide and field evaluation of riprap methods. Final Rep. No. FHWA-CA-TL-95-10. Sacramento, CA: Office of Structural Foundations, Transportation Laboratory.

Recking, A., and J. Pitlick. 2013. "Shields versus Isbash." J. Hydraul. Eng. 139 (1): 51-54. https://doi.org/10.1061/(ASCE)HY.1943-7900 .0000647 .

Reese, A. 1984. "Riprap sizing four methods." In Proc., ASCE Hydraulics Division Specialty Conf. New York: ASCE.

Richardson, E. V., and S. R. Davis. 2001. Evaluating scour at bridges. Rep. No. FHWA NHI-01-001. Washington, DC: Federal Highway Administration.

Schleiss, A. J. 2000. "Bemessung und Gestaltung von Blockwürfen an Gebirgsflüssen [Design and landscape integration of riprap in mountain rivers]." In Vol. 2 of Proc., Int. Symp. Interpraevent 2000, 351-360. Klagenfurt, Austria: Interpraevent.

Shields, A. 1936. Application of similarity principles and turbulence research to bed-load movement. Translated by W. P. Ott, and J. C. van Uchelen. Pasadena, CA: California Institute of Technology.

Simons, D. B., and F. Y. Senturk. 1992. Sediment transport technology. Fort Collins, CO: Water Resources Publications.

Stevens, M. A., D. B. Simons, and G. L. Lewis. 1976. "Safety factors for riprap protection.” J. Hydraul. Div. 102 (5): 637-655.

Stevens, M. A., D. B. Simons, and E. V. Richardson. 1984. "Riprap stability analysis." Transp. Res. Rec. 950: 209-216.

Straub, L. G. 1953. "Dredge fills closure of Missouri River at Fort Randall." In Proc., Minnesota Int. Hydraulics Convention, 61-75. Minneapolis: WMC Brown.

Ulrich, T. 1987. "Stability of rock protection on slopes." J. Hydraul. Eng. 113 (7): 879-891. https://doi.org/10.1061/(ASCE)0733-9429(1987) 113:7(879).

USACE. 1990. Hydraulic design of spillways. EM 1110-2-1603. Washington, DC: US Government Printing Office.

USACE. 1994. Hydraulic design of flood control channels. EM 1110-21601. Washington, DC: US Government Printing Office.

USBR (US Dept. of the Interior Bureau of Reclamation). 2014. Reclamation managing water in the West. Design Standard No. 13. Washington, DC: US Dept. of the Interior.

Wittler, R. J., and S. R. Abt. 1988. "Riprap design by modified safety factor method." In Proc., 1988 National Conf. on Hydraulic Engineering, 143-148. Reston, VA: ASCE. 